\title{
ON-LINE DETECTION OF DEFECTS ON FRUIT BY MACHINE VISION SYSTEMS BASED ON THREE-COLOR-CAMERAS SYSTEMS
}

\author{
Qiaobao Xu ${ }^{1}$, Xiaobo Zou ${ }^{2, *}$, Jiewen Zhao ${ }^{2}$ \\ 1 Electro-mechinery General Works Co.Ltd, Jiangsu University, Zhenjiang 2120132 \\ 2 School of Food and Biological Engineering, Jiangsu University, Zhenjiang 2120132 \\ * Corresponding author, Address: School of Food and Biological Engineering, Jiangsu \\ University, Zhenjiang 212013, Jiangsu Province, P. R. China, Tel: +86-511-88780201, \\ Fax: +86-511-88780201,Email:zou_xiaobo@ujs.edu.cn
}

Abstract: How to identify apple stem-ends and calyxes from defects is still a challenging project due to the complexity of the process. It is know that the stem-ends and calyxes could not appear at the same image. Therefore, a contaminated apple distinguishing method is developed in this article. That is, if there are two or more doubtful blobs on an apple's image, the apple is contaminated one. There is no complex imaging process and pattern recognition in this method, because it is only need to find how many blobs (including the stem-ends and calyxes) in an apple's image. Machine vision systems which based 3 color cameras are presented in this article regarding the online detection of external defects. On this system, the fruits placed on rollers are rotating while moving, and each camera which placed on the line grabs 3 images from an apple. After the apple segmented from the black background by multi-thresholds method, defect's segmentation and counting is performed on the apple's images. Good separation between normal and contaminated apples was obtained for threecamera system (94.5\%), comparing to one-camera system (63.3\%), twocamera system $(83.7 \%)$. The disadvantage of this method is that it could not distinguish different defects types. Defects of apples, such as bruising, scab, fungal growth, and disease, are treated as the same.

Keywords: Apple, defect, segmentation, imaging processing, prediction

Please use the following format when citing this chapter:

Xu, Q., Zou, X. and Zhao, J., 2009, in IFIP International Federation for Information Processing, Volume 295, Computer and Computing Technologies in Agriculture II, Volume 3, eds. D. Li, Z. Chunjiang, (Boston: Springer), pp. 2231-2238. 


\section{INTRODUCTION}

China has an annual year apple production over 20 million tons since 2000. After apples are harvested, they are transported to the packing plant to be tested for various quality attributes that determine their price and destination. The external appearance is one of the most important factors in pricing the apples. Machine vision and imaging processing techniques have been increasingly important for the fruit industry, especially when applied to quality inspection and defect sorting applications. Automated inspection of apple quality involves computer recognition of good apples and blemished apples based on geometric or statistical features derived from apple images. Nowadays, mechanical non-destructive devices for on-line measurement of weight, size and color working at high speed (several fruits/s), are common in current packinghouses. However, automating the defect sorting process is still a challenging project due to the complexity of the process. One of the biggest difficulties involved in the technology of automated machine vision inspection of fruit defects is how to distinguish the stem-end (stem cavity) and calyx (bloom bottom) from true defects such as bruises, insect damages, and blemishes.

As it may be seen, most researchers did not consider how to manage several images representing the whole surface of the fruit(Leemans, 2004). It seems that each image was treated separately and that the fruit was classified according to the worse result of the set of representative images. It is know that the stem-ends and calyxes could not appear at the same image. Therefore, if there are two or more doubtful blobs on an apple's image, the apple is contaminated one. There is no complex imaging process and pattern recognition in this method, because it is only need to find how many blobs (including the stem-ends and calyxes) in an apple's image. However, this method needs many images to cover the whole surface of the fruit. The objective of this paper investigates machine vision systems which based on 3 normal-color-cameras regarding the online detection of external defects and get the whole surface of the fruit.

\section{MATERIALS AND METHODS}

\subsection{The hardware}

The lighting and image acquisition system were designed to be adapted on an existing single row grading machine (prototype from Jiangsu Univ., China). Four lighting tubes (18 W, type 33 from Philips, Netherlands) were placed at the inner side of a lighting box while three cameras (colour 3CCD 
uc610 from Uniq, U.S.A.), two inclined at about $60^{\circ}$ and one above observed the grading line in the box, as shown in Fig. 1. The standard image treatment functions were based on the Matrox libraries (Matrox, Canada) and the other algorithms were implemented in $\mathrm{C}++$. A local network was build among the three computers in order to communicate results data. The central processing unit of each computer was a Pentium 4 (Intel, USA) clocked at 3.0GHz. The fruits placed on rollers are rotating while moving. The rotational speed of the rollers was adjusted in such a way that a spherical object having a diameter of $80 \mathrm{~mm}$ made a rotation in exactly three images. The moving speed in the range $0 \sim 15$ apples per second could be adjusted by the stepping motor. The fruit grading was achieved in seven steps: image acquisition; image preprocessing; size and shape classification; color classification; defect segmentation and recognition; three computer communication data and finally the fruit classification into quality classes.

\subsection{Image acquisition and preprocessing}

The Matrox digitizer supports trigger input, this all us to grab a frame upon external trigger. The external trigger that is composed of an emitter and an acceptor was place at the grading line as shown in fig. 1 . The three framegrabbers grab images when every roller passes through the trigger. As it mentioned above that there are three apples in the view field of each camera, therefore, nine images were grabbed from am apple as shown in fig.2.

The images in the field of view are not only the apples waiting for measurement but also including the rollers in grading line and other mechanical parts above line. Image preprocessing includes background segmentation, image de-noise, child image segmentation and sequential images processing.

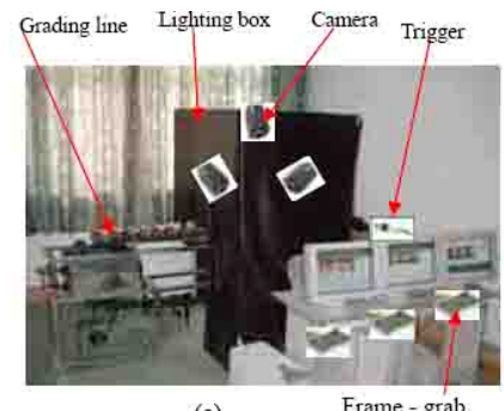

(a)

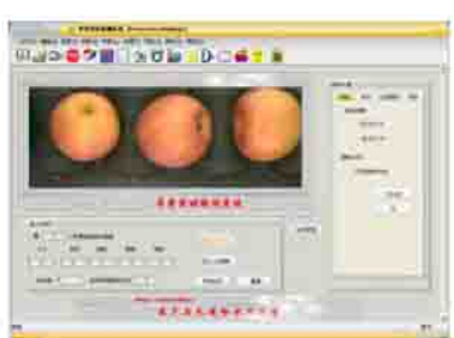

(b)

Fig. 1: Sketch map of hardware system of apple on-line detection

(a) System hardware (b) Software interface 


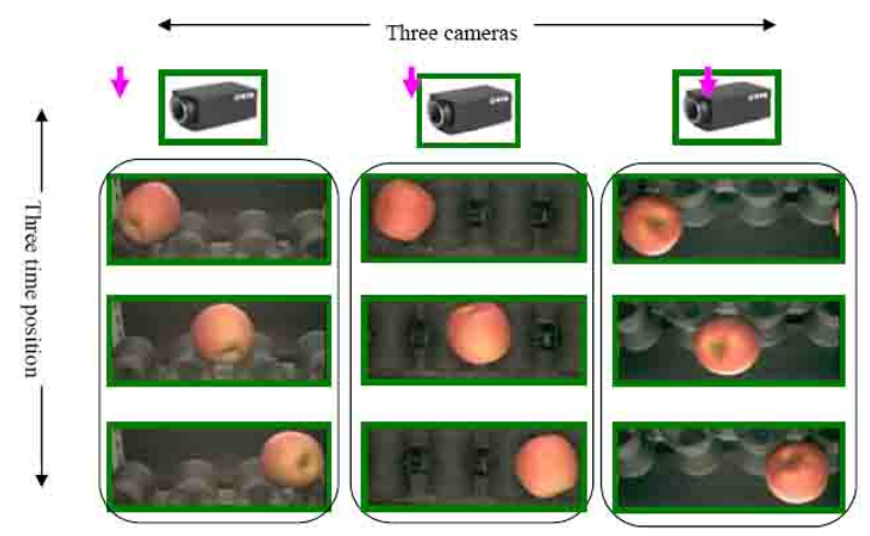

Fig.2: Trigger grab of nine images for an apple by three cameras at three position

The background is relatively complicated. To get rid of the background, multi-thresholds method was put forward. That is, the R value in RGB(redgreen-blue) and S value in HIS (hue- intensity - saturation) were taken into account. The segmentation values are as follows

$$
p(x, y)=\left\{\begin{array}{cc}
\text { background pixel : } & \mathrm{R}<90 \|(\mathrm{S}<0.20 \& \& \mathrm{R}<200) \\
\text { apple pixel : } & \text { else }
\end{array}\right\}
$$

There may still be some noises in the image after getting rid of the background, so this paper introduces medial filter to getting rid of the noise.

There are three apples waiting for measurement in the field of view at most. In order to take out one's own information of the individual apple, single apple division has become inevitable operation. The minimum enclosing rectangle of each single apple was used to divide the view image to three child images.

\subsection{Blemish (or defects) segmentation and recognition}

An image analysis scheme for accurate detection of fruit blemishes proposed by is used in this study. The detection procedure consists of two steps: initial segmentation and refinement. In the first step, blemishes are coarsely segmented out with a flooding algorithm and in the second step an active contour model, i.e. a snake algorithm, is applied to refine the segmentation so that the localization and size accuracy of detected blemishes is improved. The tests for sample apple images have shown very good results than other methods.

The blemish recognition steps are as fallowing:

First, the regions issued from the segmentation process including the defects, calyx and stem ends are hereafter called blobs. 
Second, the number of blobs is count in each single child apple image.

Third, logic distinguish method were developed. That is, since calyx and stem ends could not appear in a single child image at the same time, an apple has defects as long as any one of an apple's nine images has more than two blobs. Fig. 3 shows an example of apple images has two blobs.

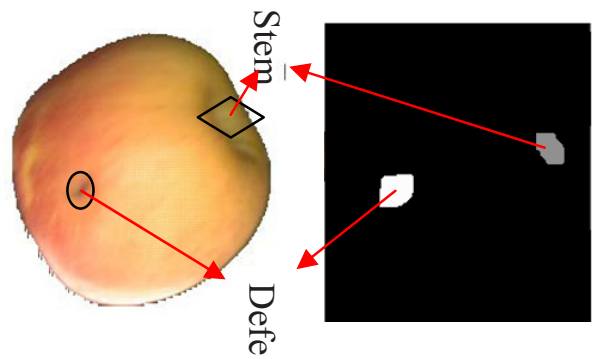

Fig.3 Precise segmentation of blob area

Fourth, three computers are used in the grading line. The apples character parameters of an apple got by each computer should be integrate into one computer. The defect detection mentioned above is all based on the three computers information. One of the three computers is server; the other two computers are customers.

Since nine image of an apple could enough cover the whole surface of the apple, any defects in the apple surface could not be omitted by this method. The disadvantage of this method is that it could not distinguish different defect types. Defects of apples, such as bruising, scab, fungal growth, and disease, are treated as the same. The fruits were then graded to reject apples if there is defect fined in the apple.

\section{$2.4 \quad$ Fruits}

All the fruits used in this experiment were selected and came from the same grower. 318 fruits were used in only one experiment and each fruit was thus presented only once to the machine, to avoid any additional bruises. The apples were classified into two classes: accepted (199 apples) and rejected (with blemish, 119 apples). 


\section{RESULTS AND DISCUSSION}

The results obtained by the three color cameras grading line are given in table1. The error rate reached $11 \%$ and most of these were accepted fruits which were classified into rejected. When these errors were analyzed, half of the errors were fruits with over-segmentation of healthy tissue and especially the tissue near the boundaries in the defect segmentation processing. The other half was due to two reasons. Firstly, spot blush on the accept apples surface is segment as defect. When there is a spot blush on the accept apple surface, the spot blush is easy to be segment as defect, and the apple is classified into rejected class. Secondly, errors were fruits with defects which were accepted. The defects were always present in the upper part of the fruits. They have almost the same appearance as the russet around the stem end and, because of the proximity in position and appearance, were probably confused with the latter.

Table. 1 The results obtained by the three color cameras grading line

\begin{tabular}{lcc}
\hline & True groups Accepted (199 apples) & Rejected(with defects, 119 apples) \\
Graded in & 169 & 5 \\
I (accepted) & 30 & 114 \\
Rejected & $15.07 \%$ & $4.2 \%$ \\
Classification error & \multicolumn{2}{c}{$11 \%$} \\
Global classification error & \multicolumn{2}{l}{} \\
\cline { 2 - 3 }
\end{tabular}

As comparing study, the results of sorting line with only one camera (the above camera) and sorting line with the two inclined camera, were shown in table 2 and table 3 . The errors of classification are rising when the cameras decline, especially for the rejected class as $4.2 \%$ for three cameras and $21.8 \%$ for one camera which are shown in table 1-3. However, at the same time, the error rates for accept class are decline slightly, as $15.07 \%$ for three cameras and $11 \%$ for one camera. This is mainly caused by the images decline as the cameras decline. A statistic test was carried out for the losing of apple's information when the different number of camera was used in the sorting line. There is $15 \%-20 \%$ of the apple's surface can not see from the 3 images which obtained by the above camera, and there is $5 \%-10 \%$ of the apple surface will be lose in the 6 images which obtained by the two inclined camera. However, the 9 images obtained by the three cameras could cover the whole surface of the apple. For the reject apples, the more images there are more opportunity to find the blemish, and the less error classification of rejected class. For the accept apples, the more images, the more spot blush on the accept apples surface will be find, and the more apples will be misclassified. The global classification error for the online sorting of the three cameras is the highest. There are little reject apples misclassified into accept ones. Compared with many former works in articles(Bennedsen, et al., 2005, Blasco, et al., 2007), several images representing the whole surface of 
the fruit are considered in this work, and the defect recognition algorithm is simpler and faster.

Table. 2 The results obtained by the two inlined color cameras grading line

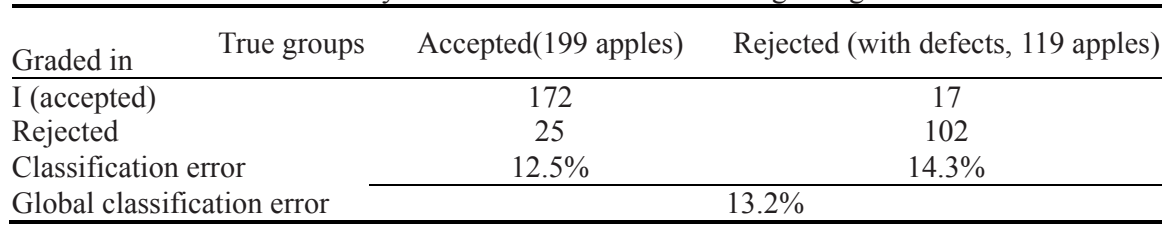

Table. 3 The results obtained by the only one camera (the above camera) grading line

\begin{tabular}{|c|c|c|c|}
\hline Graded in & True groups & Accepted ( 199 apples) & Rejected (with defects, 119 apples) \\
\hline \multicolumn{2}{|l|}{ I (accepted) } & 177 & 26 \\
\hline \multicolumn{2}{|c|}{ Rejected } & 22 & 93 \\
\hline \multicolumn{2}{|c|}{ Correct classification rate } & $11 \%$ & $21.8 \%$ \\
\hline Global corre & ct classification rate & & $15.1 \%$ \\
\hline
\end{tabular}

\section{CONCLUSION}

The grading of apples into quality classes is a complex task involving different stages. The prior step is the images acquisition, which was performed by three color CCD cameras during the motion of the fruit on an adapted machine. It was followed by a first segmentation to locate the fruits on the background and a second one to find the possible defects. Once more than one defect was finding, the apple was classified into reject class.

The correct classification rate of 318 "fuji" apples was of $89 \%$, and significantly increased, especially for the reject class. The errors were due to defects difficult to segment such as russet and bruises. Some wounds located near the stem ends were probably confused with these ends. Enhancement of the grading process should come from every stage, and particularly, from the image acquisition stage. Indeed, better light repartition and use of specific wavelengths revealing high contrast between defects and healthy tissue should probably enhance the global machine performances.

\section{ACKNOWLEDGEMENTS}

This work is supported by "Natural Science Foundation of China" and "863 high-tech fund of China" Fund. We also wish to thank many of our colleagues for many stimulating discussions in this field 


\section{REFERENCES}

Abdullah, M. Z., Mohamad-Saleh, J., Fathinul-Syahir, A. S. \&Mohd-Azemi, B. M. N. (2006). Discrimination and classification of fresh-cut starfruits (Averrhoa carambola L.) using automated machine vision system. Journal of Food Engineering 76(4): 506-523.

Bennedsen, B. S., Peterson, D. L. \&Tabb, A. (2005). Identifying defects in images of rotating apples. Computers and Electronics in Agriculture 48(2): 92-102.

Blasco, J., Aleixos, N. \&Molto, E. (2007b). Computer vision detection of peel defects in citrus by means of a region oriented segmentation algorithm. Journal of Food Engineering 81(3): 535-543.

Furfaro, R., Ganapol, B. D., Johnson, L. F. \&Herwitz, S. R. (2007). Neural network algorithm for coffee ripeness evaluation using airborne images. Applied Engineering in Agriculture 23(3): 379-387.

$\mathrm{Gu}$, X. (1994). Multi-spectrum imagery techniques quicken the sorting of agricultural products. Hongwai/Infrared (5): 41-42.

Leemans, V. D., M.-F. (2004). A real-time grading method of apples based on features extracted from defects. Journal of Food Engineering 61: 83-89.

Lefcout, A. M., Kim, M. S., Chen, Y. R. \&Kang, S. (2006). Systematic approach for using hyperspectral imaging data to develop multispectral imagining systems: Detection of feces on apples. Computers and Electronics in Agriculture 54(1): 22-35.

Mehl, P. M., Chao, K., Kim, M. \&Chen, Y. R. (2002). Detection of defects on selected apple cultivars using hyperspectral and multispectral image analysis. Applied Engineering in Agriculture 18(2): 219-226.

Throop, J. A., Aneshansley, D. J., Anger, W. C. \&Peterson, D. L. (2005). Quality evaluation of apples based on surface defects: development of an automated inspection system. Postharvest Biology and Technology 36(3): 281-290.

Wen, Z. \&Tao, Y. (1998).Dual-wavelength imaging for on-line identification of stem-ends and calyxes. Vol. 3460, 249-253 San Diego, CA, United States: The International Society for Optical Engineering.

Yang, Q. (1994). Approach to apple surface feature detection by machine vision. Computers and Electronics in Agriculture 11(2-3): 249-264. 